recommends patients with baseline bodyweight $<77 \mathrm{~kg}$ or baseline platelet count $<150 \times 10^{3} / \mu \mathrm{l}$ to initiate niraparib at $200 \mathrm{mg}$ and all other patients, at $300 \mathrm{mg}$. ISD was adopted in NORA (NCT03705156), the published pivotal study in Chinese patients, who generally have lower body weights than Western patients. While niraparib demonstrated acceptable safety in NORA, dose reduction remained essential and occurred in $59.9 \%$ of patients. This post hoc analysis aims to characterise the major TEAEs necessitating dose modification in NORA, to inform TEAE management in Chinese patients receiving niraparib.

Methodology In NORA, adults with PSROC and complete/partial response to most recent platinum-containing chemotherapy were randomised 2:1 to niraparib or placebo MT of 28-day cycles. Primary endpoint was progression-free survival (PFS). First 16 patients started treatment at $300 \mathrm{mg}$, before ISD was implemented. Dose interruption $\leq 28$ days and dose reduction were permitted. Niraparib-treated patients with stabilised dosing at Cycle 4 were analysed, with descriptive statistics summarising TEAEs and descriptive PFS analysis by dose levels at the beginning of Cycle 4.

Result(s)* During Month 1/2, platelet count decrease caused dose interruption and reduction in $32.8 \% / 36.2 \%$ and $22.0 \% /$ $23.6 \%$ of patients, respectively; neutrophil count decrease caused dose interruption and reduction in 9.0\%/12.1\% and $8.5 \% / 9.2 \%$ of patients, respectively. These percentages decreased from Month 3 and remained low through Months 4-6 (figure 1). Anaemia-related dose interruption/reduction occurred in $\leq 10 \%$ of patients. Most patients' stabilised doses at Cycle 4 were $100 \mathrm{mg}(n=74)$ or $200 \mathrm{mg}(n=78)$. These two groups had comparable baseline characteristics and showed similar PFS (figure 2) from Cycle 4 onward through 12 months, regardless of germline BRCA mutation status.
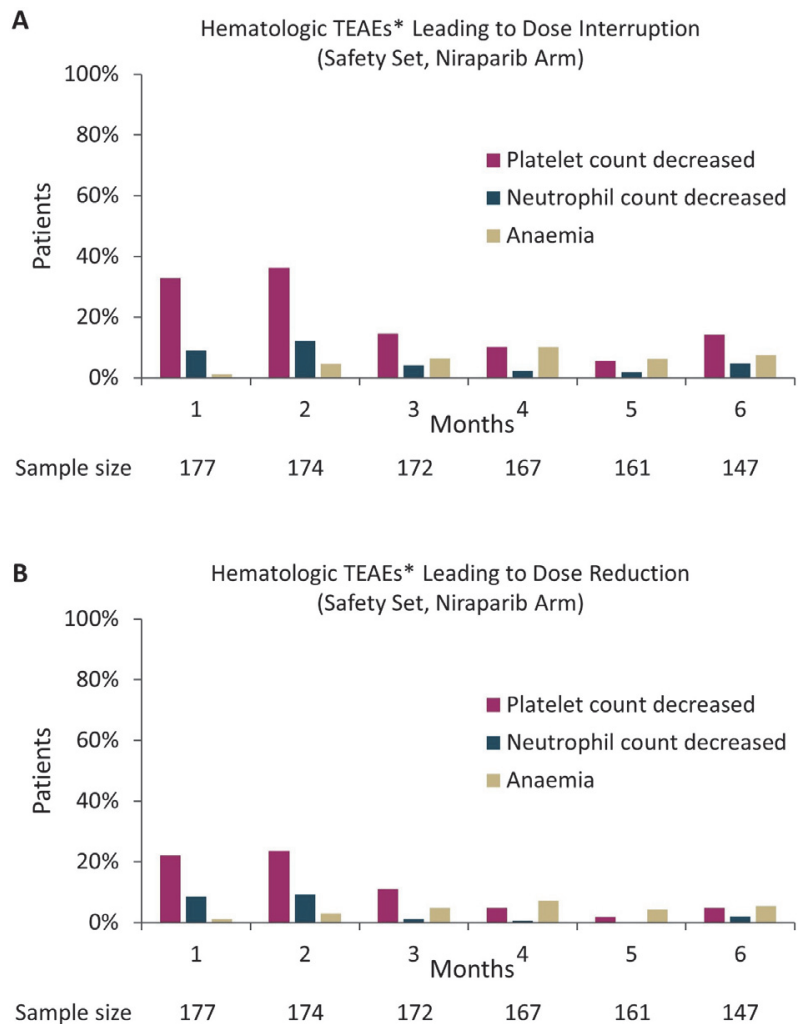

Abstract 29 Figure 1 Hematologic TEAEs Leading to niraparib dose interruption and reduction by month (Safety set)

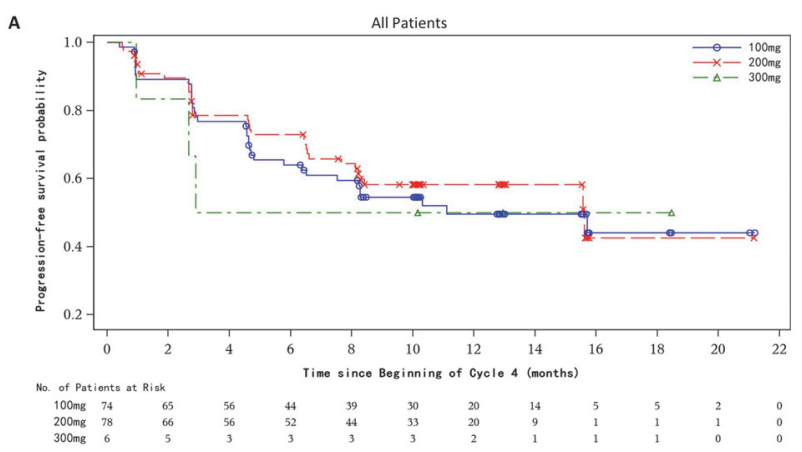

B
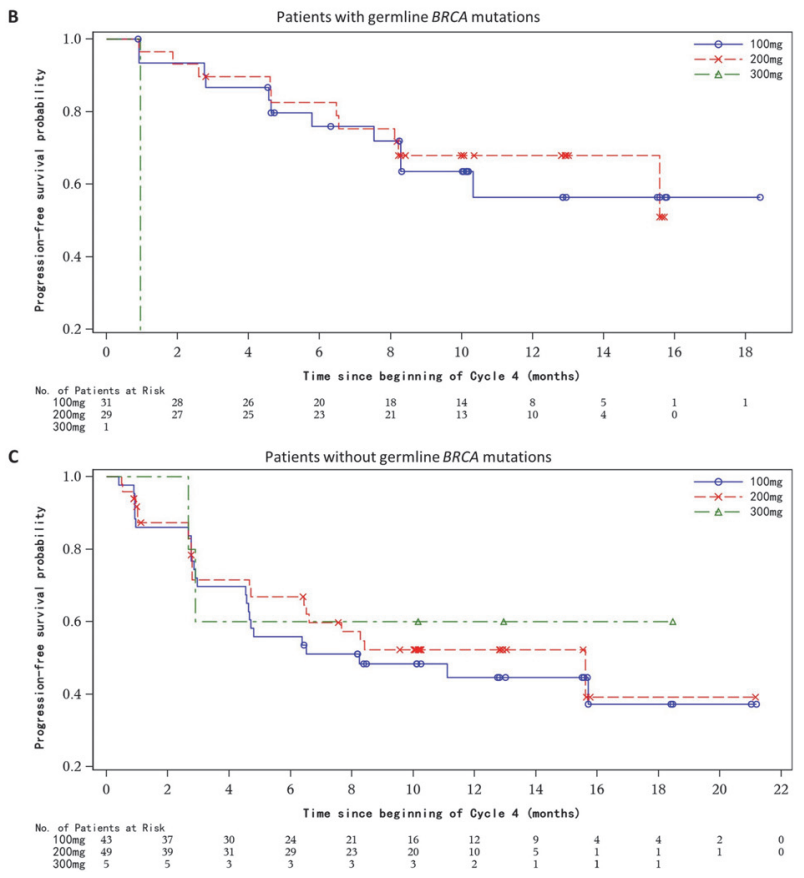

Abstract 29 Figure 2 Kaplan-meier curves for progression free survival by niraparib dose level at the beginning of cycle 4 (Intentionto-treat Set)

Conclusion* Platelet count decrease and neutrophil count decrease were the major TEAEs necessitating niraparib dose modification. Early dose modification helped maintain safety and tolerability in Chinese patients receiving niraparib MT without compromising efficacy.

\section{SURVIVAL TRENDS AND FEASIBILITY OF SURGERY IN OVARIAN CANCER PATIENTS WITH RARE METASTATIC SITES}

${ }^{1} \mathrm{M}$ Zuhdy*, ${ }^{2} \mathrm{R}$ Alghandour, ${ }^{1} \mathrm{O}$ Hamdy, ${ }^{1} \mathrm{HH}$ Metwally. 'OCMU Oncology Center Mansoura University - OCMU, Surgical oncology, Mansoura, Egypt; ${ }^{2}$ OCMU Oncology Center - Mansoura University - OCMU, Medical oncology, Mansoura, Egypt

\subsection{6/ijgc-2021-ESG0.334}

Introduction/Background* Ovarian cancer is the commonest gynaecologic malignancy in Egypt after breast cancer. Although metastasis from ovarian cancer is common, there is still sites with rarely reported deposits as non-regional nodes, bone, and brain.

Methodology We herein study retrospectively a group of patients over 7 years period recruited from data system of tertiary cancer centre. All the recruited patients suffered a rare 


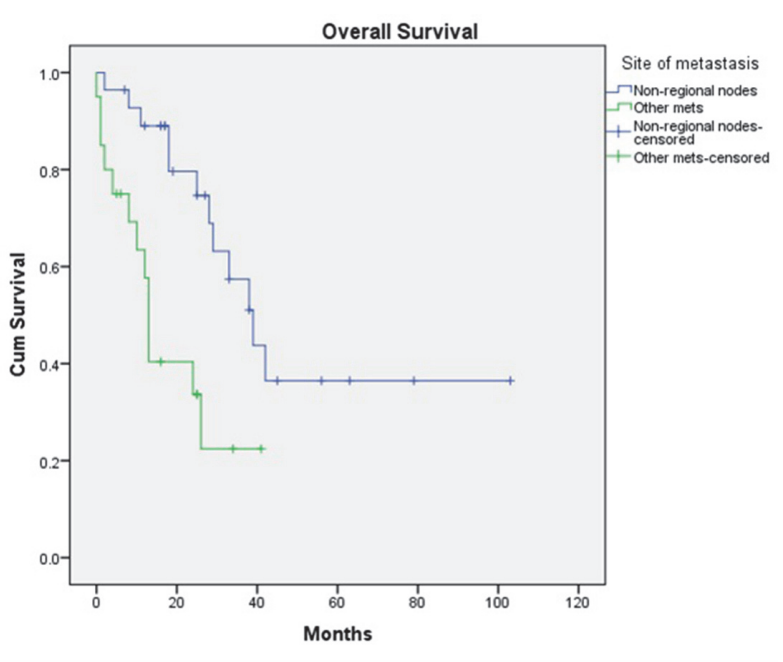

Abstract 33 Figure 1

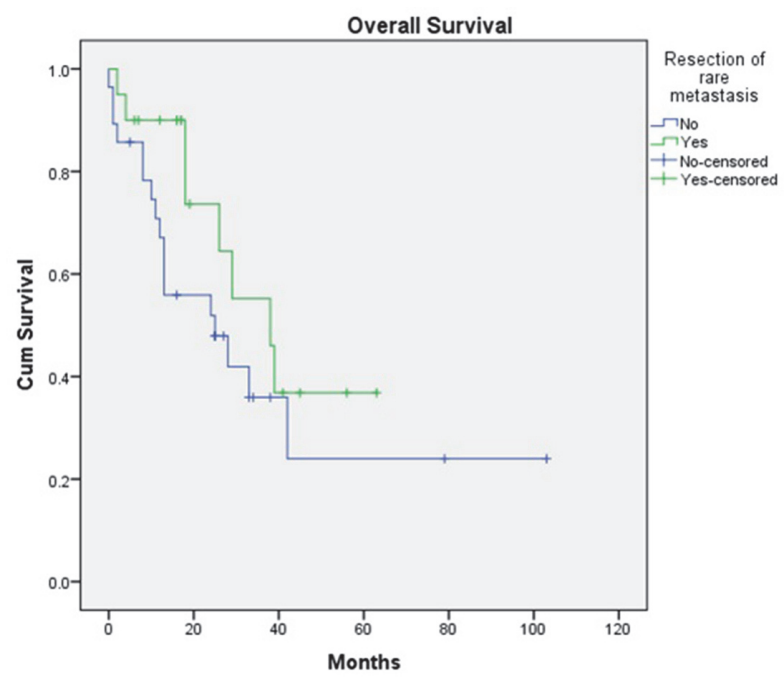

Abstract 33 Figure 2

distant metastasis from ovarian cancer (metastasis to sites other than peritoneum, liver, and lung). Demographic data, pathology, surgical treatment, and survival of these patients were analysed. Result(s)* Out of 1135 ovarian cancer patients, 48 patients with FIGO stage IV rare metastatic sites were enrolled. The most common site of rare metastasis was the non-regional lymph nodes $(56.3 \%)$, while the most common pathology was high grade serous carcinoma (87.5\%). Nearly half of the cases presented with the metastasis, while the rest developed during disease course. Interestingly, debulking was feasible in nearly a half of the patients with acceptable overall and progression free survival. Patients with non-regional nodal metastases tend to have better overall survival (39 vs 13 months $\mathrm{p}=0.003$ ) (figure 1) and progression free survival (29 vs 13 months $\mathrm{p}=$ $0.034)$ than visceral or skeletal metastasis.

Conclusion* Ovarian cancer can metastasize to rare sites. Nonregional nodes were the commonest and had better survival trends than other rare sites of metastasis. Although surgical treatment was feasible in nearly half the cases with an accepted overall and progression free survival, we could not detect survival benefit of surgical resection in these patients (figure 2).

\section{SURVIVAL OUTCOMES IN OLDER PATIENTS WITH OVARIAN CANCER DEBULKING SURGERY. IS THERE ANY DIFFERENCE BEYOND 65 YEARS OLD?}

CM Tauste Rubio*, J Zabaleta Jurío, S Aguirre Gorospe, SS Lapeña Calavia, O Tarrío Fernández, B Gastón Moreno, JC Muruzábal Torquemada. Complejo Hospitlario de Navarra, Gynecology and obstetrics, Pamplona, Spain

\subsection{6/ijgc-2021-ESG0.335}

Introduction/Background* The surgical management of advanced epithelial ovarian cancer (AEOC) is controversial in fragile subjects like elderly patients. The aim of this study was to report the global survival of primary and interval debulking surgery (PDS/IDS) over 65 years and to compare the outcomes with younger patients.

Methodology Between 2005 and 2016, 145 patients with ovarian cancer in FIGO stages IIIC or IV and PDS or IDS were included. We compared patient characteristics, type of surgery, major postoperative complications, hospital length of stay, disease free survival (DFS) and ovarall survival (OS) for patients under and over 65 years.

Result(s)* 93 patients were up to 65 years and 60 patients were over. There were no difference between the two groups for performance status, CA 125, tumors hystotypes and FIGO

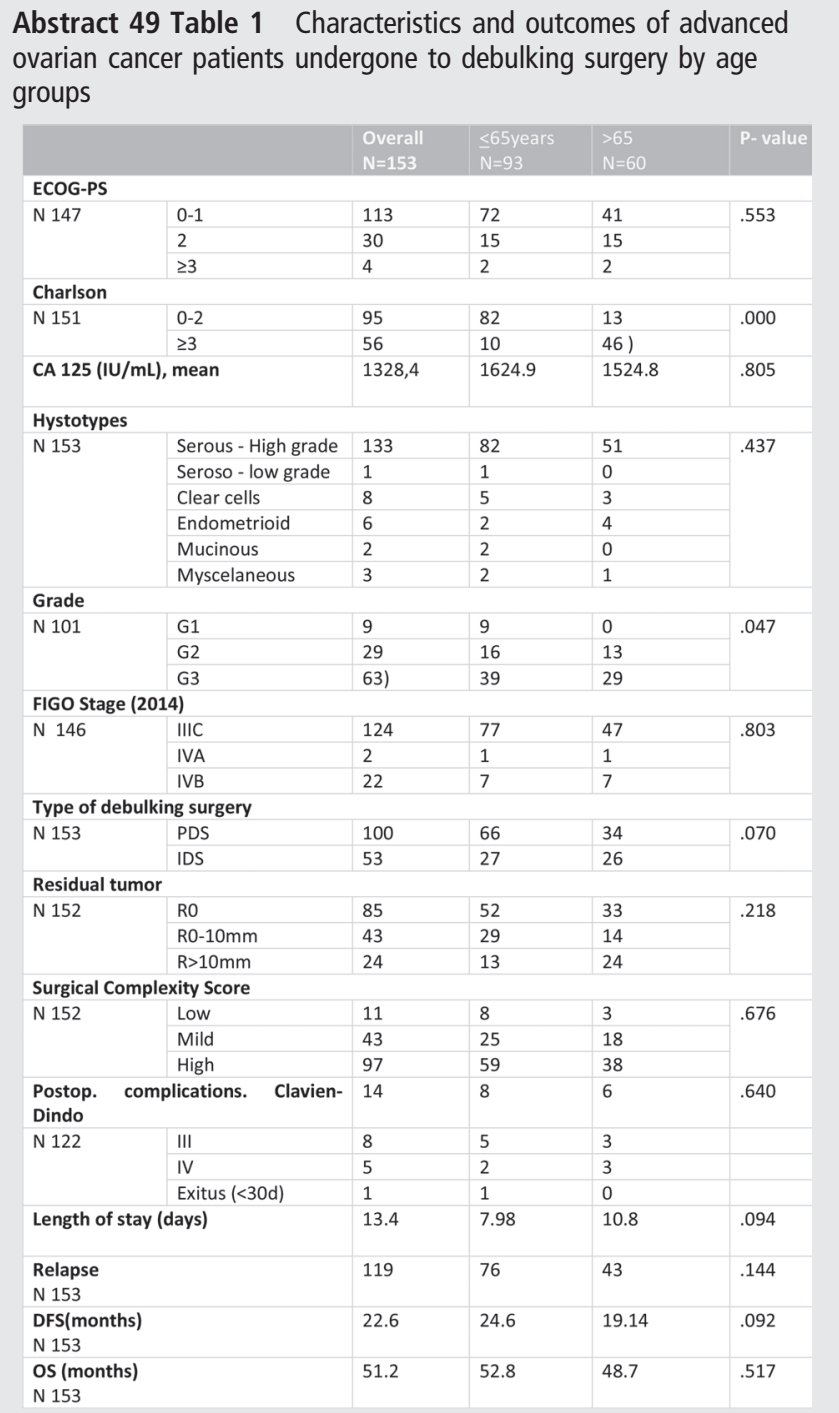

